\title{
Cranioplasty after Craniectomy in a Pediatric Population: Single-Center Experience from a Developing Country
}

\author{
Muhammad Waqas Badar Ujjan Yousaf Bashir Hadi Faizuddin Najmuddin \\ Altaf Ali Laghari Swaleha Khalid Muhammad Ehsan Bari Umar Farooq Bhatti
}

Section of Neurosurgery, The Aga Khan University Hospital Karachi, Karachi, Pakistan

\section{Keywords \\ Cranioplasty · Children · Craniectomy}

\begin{abstract}
Cranioplasty is a frequently performed procedure in neurosurgery. The pediatric population for this procedure is distinct from the adult one because of the growing skulls and thinner bones of the calvarium. A paucity of data on the outcomes of this procedure in the pediatric population has been identified repeatedly. We conducted a retrospective cohort study to investigate the outcomes in a pediatric population that underwent cranioplasty after craniectomy at our institute in a developing-world country. Our cohort showed no association of complication rate or cosmetic outcomes with the timing of cranioplasty, area of skull defect, type of implant used, or method of storage.

(c) 2016 S. Karger AG, Basel
\end{abstract}

\section{Introduction}

Cranioplasty is a frequently performed procedure in neurosurgery. It is most commonly performed after decompressive craniectomy, which is a common procedure undertaken after trauma, vascular accidents, or other causes of increased intracranial pressure. As the outcomes and long-term survival of patients after decompressive craniectomy continue to improve, so does the interest in cranioplasty, the reconstructive procedure for structural, cosmetic, and physiological rehabilitation [1]. Lately, the safety and outcomes of this procedure in children has garnered much interest, as their growing calvarium and thin bones make the pediatric population distinct from adults. A paucity of data on the outcomes of cranioplasty in children has repeatedly been identified, most recently by a systematic review conducted on the topic [1]. Moreover, populations outside of North America and Europe are not represented in the current data pool, and our retrospective cohort study aims to add to, and diversify, the literature on this topic.

\section{Materials and Methods}

We conducted a retrospective cohort study of all patients under 18 years of age who underwent cranioplasty at Aga Khan University Hospital between 2008 and 2015. All patients over 18 years of age were excluded. All patients who underwent cranioplasty after craniectomy for any indication at our hospital during the designated study period were identified using the health information
(C) 2016 S. Karger AG, Basel

E-Mail karger@karger.com www.karger.com/pne
Muhammad Ehsan Bari

Section of Neurosurgery, Department of Surgery, The Aga Khan University Hospital Stadium Road, PO Box 3500

Karachi 74800 (Pakistan)

E-Mail ehsan.bari@ aku.edu 
and management system (HIMS). The timing of cranioplasty after craniectomy is at the attending surgeon's discretion at our institution, and all craniectomized autologous bone flaps are stored at -40 to $-80^{\circ} \mathrm{C}$.

The following variables were recorded for all patients on a preformed and tested questionnaire: gender, age, reason for craniotomy, time between craniectomy and cranioplasty, site of cranioplasty, CT scan findings before and after the procedure, type of graft used, material used for fixation, complications, and the management of complications. Data analysis was performed using SPSS v. 19 software and a $p$ value $<0.05$ was taken as significant. We used $\chi^{2}$ tests and the Fischer exact test (when the expected values were less than 5) to assess the associations between categorical variables, and the Student $t$ test for assessing associations between continuous variables. Logistic regression was used to analyze the effect of different variables on the different complications observed, and adjust for confounders. Parents of the patients were asked to rate the cosmetic outcomes of the cranioplasty as satisfactory or unsatisfactory.

\section{Results}

We identified a total of 36 patients in the pediatric age group $(<18$ years of age) who underwent cranioplasty at the Aga Khan University Hospital between 2008 and 2015. The mean age of patients at the time of the procedure was 7.1 years (SD 4.97). Our cohort comprised of a majority of male patients (66.7\%). The craniplasty grafts used in our patient population were predominantly autologous bone grafts stored at -40 to $-80^{\circ} \mathrm{C}(31 / 36$ patients, $86 \%$ ), while 4 patients received methyl methacrylate and 1 patient received a titanium mesh to close the skull defect.

The mean time to cranioplasty for our patients was 15 weeks (range 4-55) and the mean duration of follow-up after cranioplasty was 79 weeks (SD 91). Trauma was the most common indication for decompressive craniectomy (61\%) followed by neoplastic lesions (9.1\%). The demographic characteristics of the cohort and the indications for craniectomy are given in Table 1. Most patients underwent a unilateral cranioplasty (77.8\%), while 7 patients (19.4\%) received a bilateral procedure. A midline cranioplasty was performed on 1 patient. Silk was the most common anchoring material applied during the procedure $(54.5 \%)$, followed by plates $(27.3 \%)$ and vicryl $(15.2 \%)$. Staples were applied to secure the graft in 1 patient. A surgical subgaleal drain was used in 16 patients $(48.5 \%)$.

There was no mortality after cranioplasty in our cohort. The overall morbidity rate for our cohort was $16.66 \%$. The most common complication was flap infection, which occurred in 3 patients (8.3\%). Other complications no-
Table 1. Demographic characteristics of the cohort and indications for craniectomy

\begin{tabular}{lr}
\hline Mean age \pm SD, years & $7.1 \pm 4.97$ \\
Gender, $n(\%)$ & \\
$\quad$ Male & $24(66.7)$ \\
$\quad$ Female & $12(33.3)$ \\
Indication for craniectomy, $n(\%)$ & \\
$\quad$ Trauma & $22(61.1)$ \\
$\quad$ Neoplastic lesion & $4(11.1)$ \\
Other & $10(27.7)$ \\
\hline
\end{tabular}

Table 2. Major complications noted in the cohort

$\begin{array}{ll}\text { Morbidity, } n(\%) & \\ \text { Flap infection } & 3(8.3) \\ \text { Wound dehiscence } & 2(5.5) \\ \text { CSF leak } & 1(2.7)\end{array}$

ticed in our cohort were wound dehiscence in 2 patients (5.5\%) and CSF leak in 1 patient. The type of implant used, reason for craniectomy, method of storage, and area of skull defect were not significantly associated with the infection rate and other complications. The morbidities recorded in the cohort are summarized in Table 2.

The parents of our patients were contacted to rate the cosmetic outcomes of cranioplasty; $72.2 \%$ were satisfied with the cosmetic outcomes, while $27.8 \%$ rated the outcomes as unsatisfactory. The time interval between craniectomy and cranioplasty, indication for craniectomy, site of cranioplasty, and area of skull defect were not significantly associated with the cosmetic outcome of cranioplasty.

\section{Discussion}

A recent systematic review identified a paucity of articles describing outcomes after cranioplasty in children and adolescents [1]. The pediatric population is considered distinct from the adult population regarding cranioplasty because of the growing calvarium and thinner bones, which predispose to complications after the procedure.

In adults, a titanium mesh with hydroxyapatite cement or acrylic as the material for cranioplasty is well tolerated. Hydroxyapatite is particularly favored because of its increased conformity to bone defects and improved osseointegration. These materials are generally avoided in
Waqas/Ujjan/Hadi/Najmuddin/Laghari/ Khalid/Bari/Bhatti 
young children, especially those less than 5 years of age, because of their growing skulls [2].

Autologous bone grafts have become the universal material of choice for pediatric cranioplasties as their osseointegration with the growing skull has been well established. The craniectomized bone flap can either be frozen at controlled temperatures, or it can be autoclaved as an alternative. In resource-poor healthcare settings, such elaborate arrangements may not be available. In such settings, insertion of the flap into the abdominal subcutaneous fat at the time of craniectomy is a viable alternative [3]. A study conducted in an adult population found no difference between infection rates of flaps that were frozen or stored in subcutaneous pockets $(p=0.23)$ [4]. Subcutaneous pockets may not be suitable for very young children because of their limited abdominal fat thickness. In our practice, all autologous flaps were stored at -40 to $-80^{\circ} \mathrm{C}$.

It was previously suggested that reinsertion of the flap after at least 6 months, and preferably after 12 months of the craniectomy, results in a lower infection rate [5]. Other studies have shown no such association $[2,3]$. More recently, this idea was challenged by Piedra et al. [6], who found that a shorter interval (less than 6 weeks) between the 2 procedures resulted in better outcomes due to less bone resorption. Our cohort yielded no significant association of adverse outcomes with the time interval between the 2 procedures. This is in keeping with the recent systematic review by Rocque et al. [1].

Rocque et al. [1] noted that a total of 11 studies comprising 441 patients have previously reported the out- comes of pediatric cranioplasties [2, 6-15]. Most studies suffer from small sample sizes. No studies from outside of North America and Europe were found. A combined infection rate of $6.8 \%$ was reported, which is similar to the flap infection rate of $8.3 \%$ found in our cohort.

Previously, 2 studies by Frassanito et al. [14] and Bowers et al. [15] have shown an association of complications after pediatric cranioplasty with patient age. It has been postulated that an increased bone turnover in very young children may be responsible for worse outcomes in this age group. Our cohort yielded no such significant association. The type of implant used, reason for craniectomy, method of storage, and area of skull defect were not significantly associated with the infection rate or other complications in our cohort.

An extensive literature review failed to yield any data on outcomes of cranioplasty in the pediatric age group from South Asia or developing countries. Our study adds to the current data pool on the subject and diversifies it by contributing outcomes from this previously unrepresented population.

\section{Conclusion}

Our study adds to the current literature on outcomes of cranioplasty in the pediatric age group. Our cohort showed no association of complication rate or cosmetic outcomes with the timing of cranioplasty, area of skull defect, type of implant used, or method of storage.

\section{References}

1 Rocque BG, Amancherla K, Lew SM, Lam S: Outcomes of cranioplasty following decompressive craniectomy in the pediatric population: A systematic review. J Neurosurg Pediatr 2013;12:120-125.

2 Josan VA, Sgouros S, Walsh AR, Dover MS, Nishikawa H, Hockley AD: Cranioplasty in children. Childs Nerv Syst 2005;21:200-204.

3 Flannery T, McConnell RS: Cranioplasty: why throw the bone flap out. Br J Neurosurg 2001; 15:518-520.

4 Inamasu J, Kuramae T, Nakatsukasa M: Does difference in the storage method of bone flaps after decompressive craniectomy affect the incidence of surgical site infection after cranioplasty? Comparison between subcutaneous pocket and cryopreservation. J Trauma 2010;68:183-187.

5 Hockley AD, Goldin JH, Wake MJC, Iqbal J: Skull repair in children. Pediatr Neurosurg 1990-1991;6:271-275.
6 Piedra MP, Thompson EM, Selden NR, Ragel BT, Guillaume DJ: Optimal timing of autologous cranioplasty after decompressive craniectomy in children: clinical article. J Neurosurg Pediatr 2012;10:268-272.

7 Blair GA, Gordon DS, Simpson DA: Cranioplasty in children. Childs Brain 1980;6:82-91.

8 Gruber R, Peter R, Hora J: The prognosis of cranioplasty following large craniectomy in children. Z Kinderchir 1988;43:375-383.

9 Pochon JP, Klöti J: Cranioplasty for acquired skull defects in children - a comparison between autologous material and methylmethacrylate 1974-1990. Eur J Pediatr Surg 1991; 1:199-201.

10 Posnick JC, Goldstein JA, Armstrong D, Rutka JT: Reconstruction of skull defects in children and adolescents by the use of fixed cranial bone grafts: long-term results. Neurosurgery 1993;32:785-791.
11 Blum KS, Schneider SJ, Rosenthal AD: Methyl methacrylate cranioplasty in children: long-term results. Pediatr Neurosurg 1997; 26:33-35

12 Taggard DA, Menezes AH: Successful use of rib grafts for cranioplasty in children. Pediatr Neurosurg 2001;34:149-155.

13 Grant GA, Jolley M, Ellenbogen RG, Roberts TS, Gruss JR, Loeser JD: Failure of autologous bone-assisted cranioplasty following decompressive craniectomy in children and adolescents. J Neurosurg 2004;100:163-168.

14 Frassanito P, et al: Complications of delayed cranial repair after decompressive craniectomy in children less than 1 year old. Acta Neurochir 2012;154.5:927-933.

15 Bowers CA, et al: Risk factors and rates of bone flap resorption in pediatric patients after decompressive craniectomy for traumatic brain injury. J Neurosurg Pediatr 2013;11.5: 526-532. 